\title{
The distribution and ecological factors of aerial algae inhabiting stoneworks in Korea
}

\author{
Mi-Ae Song ${ }^{1}$, Ok-Jin Kim ${ }^{1}$ and Ok-Min Lee ${ }^{1, *}$ \\ ${ }^{1}$ Department of Life Science, College of Natural Science, Kyonggi University, Suwon 443-760, Korea
}

The physico-chemical and environmental factors of stoneworks were examined from March 2009 to October 2010, and aerial algae living on 24 stoneworks in Korea were identified. Fifty aerial algae were found in 24 stoneworks. Of the 50 taxa, 30 taxa were cyanophytes, 16 taxa were bacillariophytes, 3 taxa were chlorophytes, and 1 taxon was a xanthophyte. Nine species, including Aphanocapsa rivularis, which is known to only live in aquatic conditions, inhabited both aquatic and aerial environments. Synechococcus aeruginosus, Scytonema coactile var. thermalis, S. coactile var. minor, Stigonema ocellatum f. ocellatum, and Oscillatoria boryana were newly recorded in Korea. As a result of a correlation analysis between algae and the physico-chemical and ecological environmental factors, the taxa were divided into the bryophyte group, the humidity group, and the dry group. Although the bryophyte group was included within the humidity group, it was separated from the dry group. Taxa in the bryophyte group including Nostoc commune which was distributed in more humid areas than those in the humidity group. However, dry group taxa including Chroococcus pallidus were distributed in lower humidity than that of the two other groups. Correlations among other environmental factors were tested to identify other factors that could substitute for humidity and light intensity. As a result, tree distance, water distance, and plant coverage were replaceable environmental factors.

Key Words: aerial algae; bacillariophytes; chlorophytes; cyanophytes; stoneworks; xanthophytes

\section{INTRODUCTION}

Aerial algae are algae that live in rock faces, earthen rocks, stone walls, and plants but not in water. Edaphophytes can also be included as aerial algae (Chung 1993). Studies on algae have mostly concentrated on aquatic algae, and studies conducted on aerial algae have mostly focused on biological pollution and preventive stone cultural heritage research (National Research Institute of Cultural Heritage 2008). Some taxa in Protococcus, Trentepholia, Cephaleuros, Scytonema, Nostoc, Schizothrix, Stigonema, Chlorococcum, and Trentepholia are known as aerial algae. According to Chung (1979), 90 taxa are listed as aerial algae, and 45 taxa were identified in Korea in Chung (1993). Additionally, 41 aerial algae taxa have been identified in Korea in studies by Chang et al. (1998), but only a few studies have been conducted on aerial algae.

In research on aerial algae habitat distribution, it was determined that cyanophytes, such as Gloeocapsa sp. and Lyngbya sp., inhabit the Muryong Royal Tomb in Gongju (Kim et al. 2001). Additionally, studies on Chlorella attached to a stone Buddha (Klochkova and Kim 2005), and aerial algae distributed on five (Lim and Lee 2008a) and eight (Kim et al. 2010) stone cultural properties have been conducted in Korea. (c) This is an Open Access article distributed under the terms of the Creative Commons Attribution Non-Commercial License (http://creativecommons.org/licenses/by-nc/3.0/) which permits unrestricted non-commercial use, distribution, and reproduction in any medium, provided the original work is properly cited.
Received January 25, 2012, Accepted September 24, 2012

*Corresponding Author

E-mail: omlee@kyonggi.ac.kr

Tel: +82-31-249-9643, Fax: +82-31-241-0860 

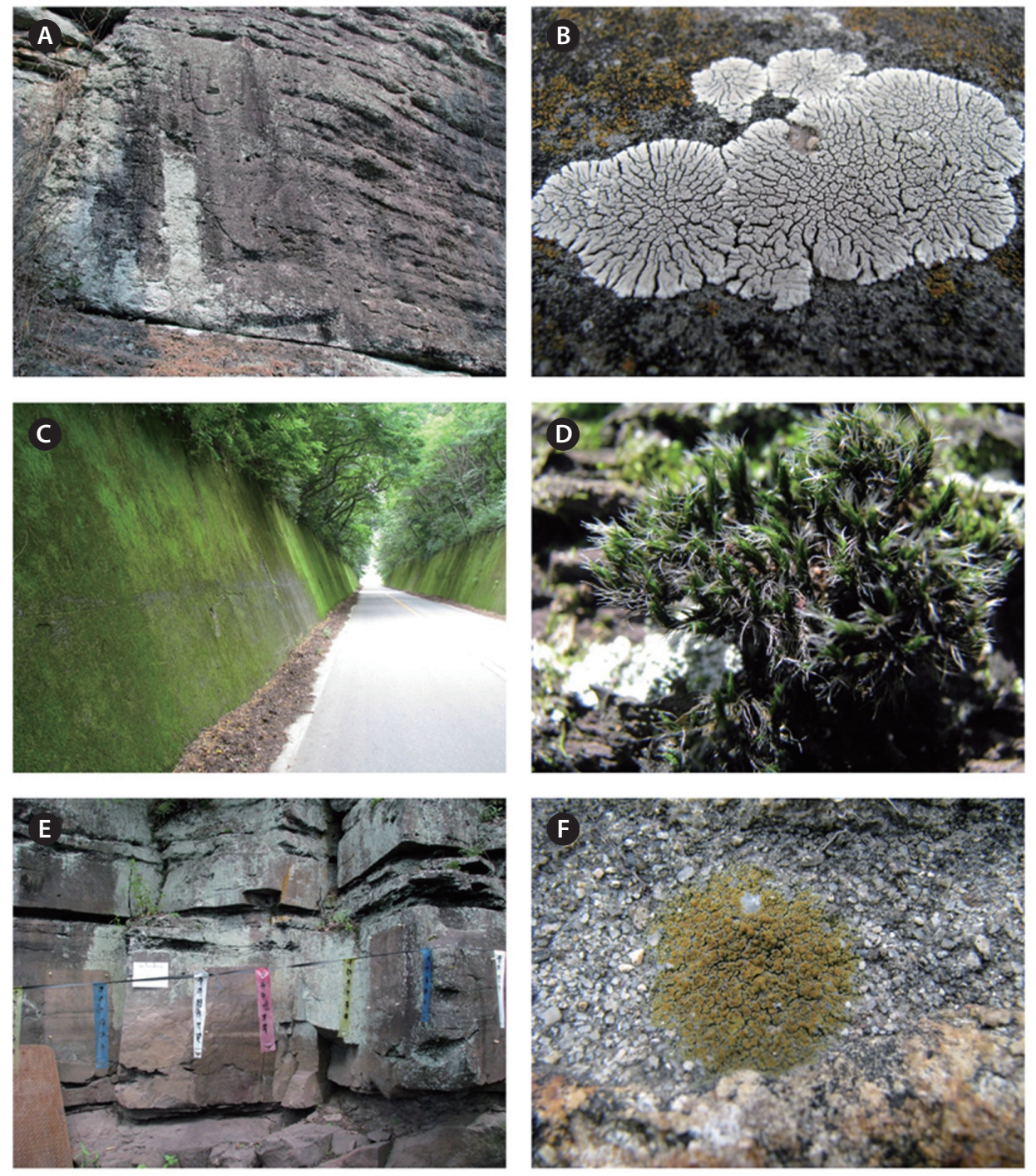

Fig. 1. Photographs the algae habitats sampled in Korea at the 24 stoneworks sites from 2009 to 2010. (A \& B) Unjusa temple (lichen and aerial algae). (C \& D) Jucksung-myeon stone wall (bryophyte). (E \& F) Yeondae small temple (lichen and aerial algae).

According to Hyvert (1972), 39 aerial algae species have been identified, and studies on the genus Synechococcus have reported that it lives endolithically with lichen in the church of Minas Gerais, Brazil (Allsopp et al. 2004). Five taxa including Coccomyxa are green algae that live on marble (Lamenti et al. 2000), and Chlorella has been discovered in the Maya ruins of Mexico (Videla et al. 2000). Research about microalgae in stoneworks located in Spain (Sarró et al. 2006) and a study of cyanophytes that inhabit a church wall located in Porto Alegre, Brazil (Crispim et al. 2004) has also been performed. The main objectives of this study were to identify aerial algae living in stoneworks and to record new species in Korea. In addition, ecological characteristics of the identified algae were investigated by analyzing the ecological factors related to their distribution.

\section{MATERIALS AND METHODS}

Collections of aerial algae were sampled at Gyeonggido, Jeolla-do, Gyeongsang-do, and Chungcheong-do on 24 stoneworks from March 2009 to October 2010 (Table 1, Fig. 1). The aerial algae samples were collected from more than one part and in every aspect of the stoneworks using a soft brush and sterilized depressor. A $5 \times 5 \mathrm{~cm}^{2}$ quadrat was used for quantitative analysis. Each sample was sealed and refrigerated in a light-tight container with 
sterilized distilled water and transferred to the laboratory (Crispim et al. 2004). Some of the samples were stored fixed in $1 \%$ formalin. Among them, the unrecorded Korean species were cultured indoors, and maintained in the algal culture collection of Kyonggi University (ACKU).

An analysis of physico-chemical environmental factors such as surface temperature of the stonework, light intensity, and humidity were conducted for all sampling dates. Temperature was measured with a stem thermometer, and surface temperature was measured with a Testo 830T1 (Testo, Lenzkirch, Germany), humidity was measured with a Testo 625 (Testo), and light intensity was assessed with a LX-1108 instrument (Lutron, Taipei, Taiwan). Temperature, humidity, and light intensity have tremendous effects on algae distribution; however, these variables largely depend on the season and time of collection. Therefore, we measured plant coverage, canopy, distance to trees, and distance to water to identify relatively stable environmental factors that could replace temperature, humidity, and light intensity (Fig. 2).

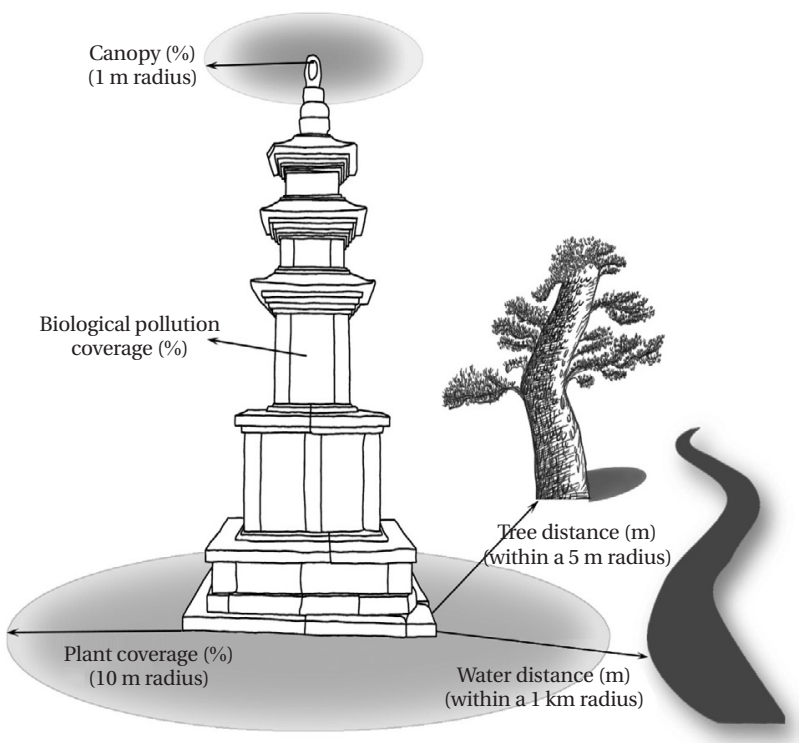

Fig. 2. Diagram of the method for measuring the environmental factors.

Table 1. Information on the 24 stoneworks from which algae were collected from March 2009 to October 2010

\begin{tabular}{|c|c|c|c|c|}
\hline Code & Stonework & Status & Date & Location \\
\hline $\mathrm{a}$ & Gwang-gyo Mountain 1-1 & $\mathrm{Na}$ & Mar 2009 & Gwang-gyo Mountain, Gyeonggi-do \\
\hline $\mathrm{b}$ & Gwang-gyo Mountain 1-2 & $\mathrm{Na}$ & & Gwang-gyo Mountain, Gyeonggi-do \\
\hline $\mathrm{c}$ & Gwang-gyo Mountain 1-3 & $\mathrm{Na}$ & & Gwang-gyo Mountain, Gyeonggi-do \\
\hline $\mathrm{d}$ & Gwang-gyo Mountain 2-1 & $\mathrm{Na}$ & & Gwang-gyo Mountain, Gyeonggi-do \\
\hline $\mathrm{e}$ & Gwang-gyo Mountain 2-3 & $\mathrm{Na}$ & & Gwang-gyo Mountain, Gyeonggi-do \\
\hline $\mathrm{f}$ & Gwang-gyo Mountain 3-1 & $\mathrm{Na}$ & & Gwang-gyo Mountain, Gyeonggi-do \\
\hline $\mathrm{g}$ & Gwang-gyo Mountain 3-2 & $\mathrm{Na}$ & & Gwang-gyo Mountain, Gyeonggi-do \\
\hline $\mathrm{h}$ & Gwang-gyo Mountain 3-3 & $\mathrm{Na}$ & & Gwang-gyo Mountain, Gyeonggi-do \\
\hline $\mathrm{i}$ & Unjusa Temple A1 & MH & Apr 2009 & Unjusa Temple, Jeollanam-do \\
\hline j & Unjusa Temple A4 & MH & & Unjusa Temple, Jeollanam-do \\
\hline $\mathrm{k}$ & Unjusa Temple A5 & MH & & Unjusa Temple, Jeollanam-do \\
\hline 1 & Unjusa Temple A6 & MH & & Unjusa Temple, Jeollanam-do \\
\hline $\mathrm{m}$ & Unjusa Temple A10 & MH & & Unjusa Temple, Jeollanam-do \\
\hline $\mathrm{n}$ & Unjusa Temple stone wall 1 & MH & Jul 2009 & Unjusa Temple, Jeollanam-do \\
\hline o & Unjusa Temple stone wall 2 & MH & & Unjusa Temple, Jeollanam-do \\
\hline $\mathrm{p}$ & Yeondae small temple A-1 & MH & Aug 2009 & Yeongyang-gun, Gyeongsangbuk-do \\
\hline $\mathrm{q}$ & Yeondae small temple A-2 & MH & & Yeongyang-gun, Gyeongsangbuk-do \\
\hline $\mathrm{r}$ & Yeondae small temple A-3 & MH & & Yeongyang-gun, Gyeongsangbuk-do \\
\hline $\mathrm{s}$ & Yeondae small temple B-1 & $\mathrm{MH}$ & & Yeongyang-gun, Gyeongsangbuk-do \\
\hline $\mathrm{t}$ & Yeondae small temple B-2 & $\mathrm{MH}$ & & Yeongyang-gun, Gyeongsangbuk-do \\
\hline $\mathrm{u}$ & Yeondae small temple B-3 & $\mathrm{MH}$ & & Yeongyang-gun, Gyeongsangbuk-do \\
\hline $\mathrm{v}$ & Stone wall 1 of Jucksung-myeon, Danyang-gun & $\mathrm{MH}$ & Jul 2010 & Danyang-gun, Chungcheongbuk-do \\
\hline $\mathrm{w}$ & Stone wall 2 of Jucksung-myeon, Danyang-gun & MH & & Danyang-gun, Chungcheongbuk-do \\
\hline $\mathrm{x}$ & Stone wall in Pyeongchang-gun & MH & Oct 2010 & Pyeongchang-gun, Gangwon-do \\
\hline
\end{tabular}

$\mathrm{Na}$, natural stone; $\mathrm{MH}$, modified for human use. 
Plant coverage was considered the distribution of woods within a $10 \mathrm{~m}$ radius of the stoneworks as a percentage. The number of trees screening light was measured within a $1 \mathrm{~m}$ radius of the stoneworks as a percentage to assess the canopy. Tree distance was considered the closest distance to a tree within a $5 \mathrm{~m}$ radius. The water distance was the closest distance to a river or lake within a 1,000 m radius. Biological pollution coverage was calculated by measuring the bryophyte area using AutoCAD 2008 (Autodesk Inc., San Rafael, CA, USA). The taxonomic classification system was based on Hirose et al. (1977), and aerial algae were identified referring to Prescott (1973), Prescott et al. (1972, 1977, 1981, 1982), Chung (1993), John et al. (2002), and Wehr and Sheath (2003). Dominant species were analyzed by relative abundance. The collected samples were examined under a light microscope $(400-1,000 \times)$ to determine the total number and the dominant species. Light microscopy was carried out using an Olympus BX41 microscope (Olympus, Tokyo, Japan) equipped with Nomarski differential interference optics. Species newly recorded in Korea were illustrated using a drawing attachment together with light microscope photographs. SPSS version 12.0 (SPSS Inc., Chicago, IL, USA) was used to analyze the relationships between aerial algae and the environmental factors.

\section{RESULTS}

Table 2 shows the results of the physico-chemical, and ecological environmental factors of the stoneworks where the aerial algae was found. The air temperature

Table 2. The physico-chemical and ecological environmental factors at 24 stoneworks from March 2009 to October 2010

\begin{tabular}{|c|c|c|c|c|c|c|c|c|c|c|}
\hline \multirow{2}{*}{ Site } & \multirow{2}{*}{$\begin{array}{l}\text { Cov } \\
(\%)\end{array}$} & \multirow{2}{*}{$\begin{array}{l}\text { Can } \\
(\%)\end{array}$} & \multirow{2}{*}{$\begin{array}{l}\text { TD } \\
(\mathrm{m})\end{array}$} & \multirow{2}{*}{$\begin{array}{l}\text { WD } \\
\text { (m) }\end{array}$} & \multirow{2}{*}{$\begin{array}{c}\mathrm{T} \\
\left({ }^{\circ} \mathrm{C}\right)\end{array}$} & \multirow{2}{*}{$\begin{array}{l}\text { ST } \\
\left({ }^{\circ} \mathrm{C}\right)\end{array}$} & \multirow{2}{*}{$\begin{array}{c}\mathrm{Hu} \\
(\%)\end{array}$} & \multirow{2}{*}{$\begin{array}{c}\mathrm{L} \\
(\mathbf{l u x})\end{array}$} & \multicolumn{2}{|c|}{ BPC $(\%)$} \\
\hline & & & & & & & & & $\mathrm{Br}$ & Li \& A \\
\hline $\mathrm{a}$ & 20 & 70 & 5 & 6 & 18.2 & 17.5 & 41.4 & 3,915 & 0 & 9.9 \\
\hline $\mathrm{b}$ & 20 & 70 & 5 & 6 & 18.2 & 17.5 & 41.4 & 3,922 & 0 & 69.3 \\
\hline $\mathrm{c}$ & 20 & 70 & 5 & 6 & 18.2 & 17.5 & 41.4 & 3,950 & 0 & 72.8 \\
\hline d & 60 & 30 & 0.6 & 30 & 12.2 & 7.5 & 58.7 & 162 & 1.2 & 29.3 \\
\hline $\mathrm{e}$ & 60 & 30 & 0.5 & 25 & 12.2 & 8.5 & 58.7 & 202 & 7.0 & 43.6 \\
\hline $\mathrm{f}$ & 30 & 60 & 0.3 & 50 & 17.5 & 15.0 & 70.7 & 1,387 & 0 & 9.7 \\
\hline g & 80 & 50 & 0 & 70 & 21.2 & 15.0 & 59.3 & 3,071 & 0 & 52.1 \\
\hline $\mathrm{h}$ & 10 & 80 & 2 & 50 & 20.1 & 15.5 & 65.7 & 2,617 & 0 & 86.1 \\
\hline $\mathrm{i}$ & 30 & 2 & 5 & 1,000 & 10.2 & 7.5 & - & - & 0 & 100 \\
\hline $\mathrm{j}$ & 10 & 0 & 5 & 1,000 & 11.1 & 7.5 & - & - & 0 & 100 \\
\hline $\mathrm{k}$ & 0 & 0 & 5 & 1,000 & 10.2 & 7.5 & - & - & 0 & 97.5 \\
\hline 1 & 10 & 0 & 3 & 1,000 & 10.2 & 7.5 & - & - & 0 & 96.3 \\
\hline $\mathrm{m}$ & 30 & 0 & 3 & 1,000 & 10.4 & 7.5 & - & - & 0 & 99.0 \\
\hline $\mathrm{n}$ & 80 & 95 & 0.1 & 0.1 & 27.2 & 23.5 & 85 & 2,500 & 16.2 & 36.3 \\
\hline o & 80 & 0 & 0.1 & 0.1 & 28.9 & 27.5 & 84.2 & 7,880 & 1.8 & 55.7 \\
\hline $\mathrm{p}$ & 50 & 60 & 0 & 1,000 & 26.7 & 28 & 80.4 & 18,500 & 1.3 & 95.5 \\
\hline$q$ & 50 & 80 & 0 & 1,000 & 26.7 & 30 & 80.4 & 18,500 & 0 & 94.2 \\
\hline $\mathrm{r}$ & 50 & 80 & 0 & 1,000 & 26.7 & 29 & 70.2 & 39,000 & 40.6 & 39.0 \\
\hline $\mathrm{s}$ & 50 & 80 & 0 & 1,000 & 25 & 23 & 73 & 1,957 & 42.7 & 51.9 \\
\hline $\mathrm{t}$ & 50 & 80 & 0 & 1,000 & 25 & 23.5 & 80 & 3,950 & 0 & 96.4 \\
\hline $\mathrm{u}$ & 50 & 80 & 0 & 1,000 & 25 & 22.5 & 90 & 2,100 & 70.5 & 23.8 \\
\hline $\mathrm{v}$ & 70 & 100 & 0 & 50 & - & 21.5 & - & - & 99.0 & 1.0 \\
\hline $\mathrm{w}$ & 70 & 60 & 0 & 50 & - & - & - & - & 62.0 & 38.0 \\
\hline $\mathrm{x}$ & 40 & 0 & 0.1 & 0.3 & 14.6 & 13.0 & - & - & 0 & 59.6 \\
\hline
\end{tabular}

Cov, plant coverage (\%); Can, canopy (\%); TD, tree distance $(\mathrm{m}) ; \mathrm{WD}$, water distance $(\mathrm{m}) ; \mathrm{T}$, temperature $\left({ }^{\circ} \mathrm{C}\right) ; \mathrm{ST}$, surface temperature $\left({ }^{\circ} \mathrm{C}\right) ; \mathrm{Hu}, \mathrm{hu}-$ midity (\%); L, light intensity (lux); BPC, biological pollution coverage (\%); BR, bryophytes; Li, lichens; Al, aerial algae. 
was $10.2-29.9^{\circ} \mathrm{C}$ and surface temperature was $7.5-30.0^{\circ} \mathrm{C}$. Humidity was $41.4-90.0 \%$. The light intensity values were the highest at the Yeondae small temple A-3 (39,000 lux), and the lowest were recorded at Gwang-gyo mountain 2-1 (162 lux). Due to strong wind, measuring the humidity, and light intensity at the Unjusa Temple, the stone wall at Jucksung-myeon, Danyang-gun, and the stone wall at Pyeongchang-gun was difficult. Plant coverage at the three sites including Gwang-gyo Mountain 3-2 had the highest value at $80 \%$, whereas the Unjusa Temple A5 was not affected by plant coverage with a value of $0 \%$. Stone wall 1 at Jucksung-myeon, Danyang-gun, which was a site where light was screened by trees, had a canopy value of $100 \%$. Five sites, including Unjusa Temple A4, had a $0 \%$ canopy value. Tree distance ranged from 0 to $5 \mathrm{~m}$, indicating that most sites were affected by trees. Water distance values were $0.1-1,000 \mathrm{~m}$.

Four phyla, 4 classes, 8 orders, 4 suborders, 13 families, 23 genera, 46 species, 3 varieties, and 1 forma were collected and identified as aerial algae (Table 3). Cyanophytes were the most dominant taxa, with 3 orders, 2 suborders, 5 families, 10 genera, 26 species, 3 varieties, and 1 forma. Only one species, Botryococus braunii, was considered a xanthophyte, and bacillariophytes had 2 orders, 2 suborders, 5 families, 9 genera, and 16 species. Chlorophytes had 2 orders, 2 families, 2 genera, and 3 species.

Stone wall 1 at Jucksung-myeon and Danyang-gun had the most taxa among the stoneworks with 21 taxa including Aphanocapsa elachista. Gwang-gyo Mountain 3-1 had only one taxon, Chroococcus varius (CHVA). C. varius had the most varied growth range, appearing in 21 different stoneworks including Gwang-gyo Mountain 1-2. Protococcus viridis, which appeared most frequently in Lim and Lee (2008b), was found at five sites including Gwanggyo Mountain 1-1.

The habitats of all taxa were evaluated after reviewing other studies (Table 3). Five taxa, including Synechococcus aeruginosus, were newly recorded in Korea. Among the 50 taxa, 37 taxa including Aphanocapsa elachista are known to inhabit aerial and aquatic environments. Additionally, 3 taxa, including Chroococcus bituminosus, were distributed only in aerial environments.

\section{Phylum Cyanophyta \\ Class Cyanophyceae \\ Order Chroococcales \\ Family Chroococcaceae \\ Synechococcus aeruginosus Nageli}

Cell long and cylindrically shaped, with a width of 6-16 $\mu \mathrm{m}$ and length of 13-30 $\mu \mathrm{m}$. Light blue-green color, with many granules; lives in freshwater and hot springs (Hirose et al. 1977).

Synechococcus aeruginosus (SYAE) lives in Sola lake in Taba Egypt (Badawy et al. 1999). In Korea, one species, Synechococcus lividus, was identified under the genus Synechococcus (Chung 1993). That study revealed that $S$. lividus lives on the surfaces of stone walls inhabited by bryophytes. The tree distance from the stoneworks was 0 $\mathrm{m}$, with $50-70 \%$ plant coverage, which was probably due to its relatively high water composition.

Sites of collection. $t, \mathrm{u}, \mathrm{v}$ (Figs 3E \& 4A).

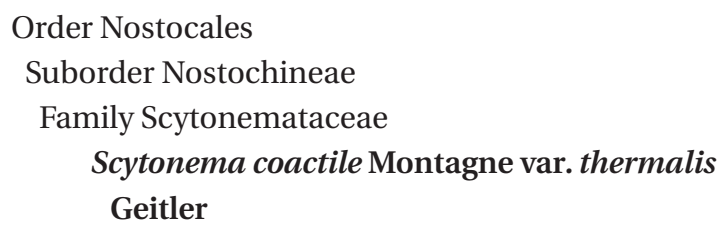

Cell olive or purple-green in color. Main axis pseudobranched and filamentous. Sheaths have no layered structure or color. Cell is (11)-15-18 $\mu \mathrm{m}$ in diameter and becomes narrower at the tip. Trichome is cylindrical with width of 9-11 $\mu \mathrm{m}$ and length of 7-20 $\mu \mathrm{m}$. Heterocysts are cylindrical, and it inhabits hot springs (Hirose et al. 1977).

Scytonema coactile var. thermalis (SCTH) has been found in Japan (Hirose et al. 1977), and we found that it lives on stone. Water distance was $>1,000 \mathrm{~m}$, and tree distance was 0-5 m.

Sites of collection. k, p, r, s, u (Figs 3A \& 4C).

\section{Order Nostocales \\ Suborder Nostochineae \\ Family Scytonemataceae}

Scytonema coactile Montagne var. minor Wille

Cell is thick and $1 \mathrm{~mm}$ in width. Pseudo-branching mostly originates from main axis. It has a yellow-brown colored sheath without a layered structure, and a diameter of 14-16 $\mu \mathrm{m}$. Trichomes are cylindrical with no joints; width is $8-10 \mu \mathrm{m}$. Heterocysts are cylindrical or square shaped, and it inhabits hot springs (Hirose et al. 1977).

Scytonema coactile var. minor (SCMI) is known to inhabit Japan and China; however, only S. crispum and $S$. myochorus has been identified locally (Chung 1993). In this study, S. coactile var. minor appeared at 10 sites including Gwang-gyo Mountain 1-1 and seemed to be affected by trees. 
Table 3. A list of aerial algae and their habitats collected at 24 stoneworks from March 2009 to October 2010

\begin{tabular}{|c|c|c|c|c|c|c|c|c|}
\hline \multicolumn{2}{|l|}{ Species } & \multicolumn{6}{|c|}{ a b c d e f ghijklmnopqrst $u$ v w $x$} & \multirow[t]{2}{*}{ Habitats } \\
\hline Phylum & Cyanophyta & & & & & & & \\
\hline Class & Cyanophyceae & & & & & & & \\
\hline Order & Chroococcales & & & & & & & \\
\hline \multirow[t]{12}{*}{ Family } & Chroococcaceae & & & & & & & \\
\hline & Aphanocapsa elachista & & & & & & + & $\begin{array}{l}\text { W (Chung 1993, John et al. 2002), } \\
\text { R (Lim and Lee 2008a) }\end{array}$ \\
\hline & A. grevillei & & & & & & $+\quad+$ & W, R, S (John et al. 2002) \\
\hline & A. pulchra & & & & & & $+\quad++$ & W (Chung 1993), S (Tiwari 1972) \\
\hline & A. rivularis & & & & & & ++ & W (Chung 1993) \\
\hline & Aphanothece naegelii & & & & & & ++ & $\begin{array}{l}\text { W, R, M (Chung 1993), S (Tiwari } \\
\text { 1972) }\end{array}$ \\
\hline & Chroococcus bituminosus & & & & & & + & R (Lim and Lee 2008a) \\
\hline & C.minutus & + & & +++ & + & & + & $\begin{array}{l}\text { W (John et al. 2002), R (Lim and } \\
\text { Lee 2008a) }\end{array}$ \\
\hline & C. pallidus & & & + & + & & & $\begin{array}{l}\text { W (Chung 1993), R (Gomez et al. } \\
\text { 2003) }\end{array}$ \\
\hline & C. varius & + & + & +++++++ & ++ & +++ & ++++ & $\begin{array}{l}\text { W (Naz et al. 2004b), R (Lim and } \\
\text { Lee 2008a, 2008b, Uher 2008) }\end{array}$ \\
\hline & Synechococcus aeruginosus $^{\mathrm{a}}$ & & & & & & +++ & $\begin{array}{l}\text { W, M (John et al. 2002), R (Bell- } \\
\text { inzoni et al. 2003) }\end{array}$ \\
\hline & Synechocystis aquatilis & & & + & ++ & & + & $\begin{array}{l}\text { W (Chung 1993), R (Lim and } \\
\text { Lee } 2008 a, 2008 b), \text { S (Naz et al. } \\
2004 b \text { ) }\end{array}$ \\
\hline Order & Nostocales & & & & & & & \\
\hline Suborder & Nostochineae & & & & & & & \\
\hline \multirow[t]{4}{*}{ Family } & Nostocaceae & & & & & & & \\
\hline & Anabaena circinalis & & & & & & + & $\begin{array}{l}\text { W (John et al. 2002), S (Ghadai et } \\
\text { al. 2010) }\end{array}$ \\
\hline & Nostoc commune & & & & & & + & $\begin{array}{l}\text { W, S, M (John et al. 2002), R (Go- } \\
\text { mez et al. 2003) }\end{array}$ \\
\hline & N. microscopicum & & & & & & ++ & $\begin{array}{l}\text { W (John et al. 2002), R (Gomez } \\
\text { et al. 2003, Uher 2008), M (West } \\
\text { and Fritsch 1972) }\end{array}$ \\
\hline \multirow[t]{4}{*}{ Family } & Scytonemataceae & & & & & & & \\
\hline & Scytonema coactile var. minor ${ }^{\mathrm{a}}$ & ++ & + & $++\quad++$ & + & & ++ & W (Hirose et al. 1977) \\
\hline & S. coactile var. thermalis ${ }^{\mathrm{a}}$ & & & + & + & ++ & + & W (Hirose et al. 1977) \\
\hline & S. crispum & & & & & & + & $\begin{array}{l}\text { W (John et al. 2002), R (Lim and } \\
\text { Lee 2008a) }\end{array}$ \\
\hline Suborder & Oscillatoriineae & & & & & & & \\
\hline \multirow[t]{10}{*}{ Family } & Oscillatoriaceae & & & & & & & \\
\hline & Lyngbya aerugineo-coerulea & + & & & & & & W, S (Chung 1993) \\
\hline & L. birgei & & & & & + & & W, S (Chung 1993) \\
\hline & Oscillatoria boryana ${ }^{\mathrm{a}}$ & & & & & + & & W (Naz et al. 2004a) \\
\hline & O. chlorina & & + & & & & ++ & W, S (Chung 1993) \\
\hline & O. geminata var. subphurea & & & & & & + & W (Chung 1993) \\
\hline & O. iwanoffiana & & & & & & + & $\begin{array}{l}\text { W (Chung 1993, Hirose et al. } \\
\text { 1977) }\end{array}$ \\
\hline & O. limosa & & & + & & & & $\begin{array}{l}\text { W (Chung 1993), S (John et al. } \\
\text { 2002) }\end{array}$ \\
\hline & O. okeni & & + & & & & & $\begin{array}{l}\text { W (Chung 1993, Naz et al. } \\
\text { 2004a), S (Tiwari 1972) }\end{array}$ \\
\hline & O. sancta & & & & & & + & $\begin{array}{l}\text { W (Chung 1993, Naz et al. } \\
\text { 2004a), S (John et al. 2002) }\end{array}$ \\
\hline
\end{tabular}


Table 3. Continued

\begin{tabular}{|c|c|c|c|c|c|c|c|c|c|c|}
\hline \multirow[t]{3}{*}{ Species } & & \multicolumn{8}{|c|}{ 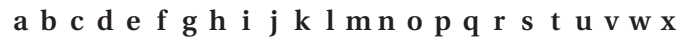 } & \multirow{2}{*}{$\begin{array}{l}\text { Habitats } \\
\text { W (Chung 1993, John et al. 2002, } \\
\text { Naz et al. 2004a), S (Naz et al. } \\
\text { 2004a) }\end{array}$} \\
\hline & O. terebriformis & & & & & & & + & & \\
\hline & O. uncinata & & & & & & & & + & $\begin{array}{l}\text { W (Chung 1993), S (Cavacini } \\
\text { 2001) }\end{array}$ \\
\hline Order & Stigonematales & & & & & & & & & \\
\hline \multirow[t]{3}{*}{ Family } & Stigonemaceae & & & & & & & & & \\
\hline & Stigonema mamillosum & & & & & & & + & & $\begin{array}{l}\text { W (Chung 1993), R (Chung 1993, } \\
\text { John et al. 2002) }\end{array}$ \\
\hline & S. ocellatum f. ocellatum ${ }^{\mathrm{a}}$ & + & + & ++ & ++++ & ++ & & +++ & + & W, R, S, M (John et al. 2002) \\
\hline Phylum & Chrysophyta & & & & & & & & & \\
\hline Class & Bacillariophyceae & & & & & & & & & \\
\hline Order & Pennales & & & & & & & & & \\
\hline Suborder & Raphidineae & & & & & & & & & \\
\hline \multirow[t]{9}{*}{ Family } & Naviculaceae & & & & & & & & & \\
\hline & Cymbella delicatula & & & & & & + & & & W (Chung 1993) \\
\hline & C. sillesiaca & & & & + & + & + & & & $\begin{array}{l}\text { W (Chung 1993), R (Poulíčková } \\
\text { and Hašler 2007) }\end{array}$ \\
\hline & Gomphonema acuminatum & & & & & & + & & & W (Chung 1993) \\
\hline & G. parvulum & & & & & & + & & & $\begin{array}{l}\text { W (Chung 1993), R (Poulíčková } \\
\text { and Hašler 2007) }\end{array}$ \\
\hline & Navicula contenta & & + & & & + & & & ++ & $\begin{array}{l}\text { W (Chung 1993), R (Poulíčková } \\
\text { and Hašler 2007) }\end{array}$ \\
\hline & N. cryptocephala & & & & & & & & + & $\begin{array}{l}\text { W (Chung 1993), R (Lim and Lee } \\
2008 a \text { ) }\end{array}$ \\
\hline & N. goeppertiana & + & + & ++ & +++ & +++ & & + & +++ & $\begin{array}{l}\text { W (Chung 1993), R (Poulíčková } \\
\text { and Hašler 2007) }\end{array}$ \\
\hline & N. subminuscula & & & + & & + & & & & W (Chung 1993) \\
\hline \multirow[t]{2}{*}{ Family } & Diatomaceae & & & & & & & & & \\
\hline & Pinnularia borealis & & + & & ++++ & ++ & & + & +++ & $\begin{array}{l}\text { W (Chung 1993), R (Poulíčková } \\
\text { and Hašler 2007, Uher 2008), S } \\
\text { (Cavacini 2001) }\end{array}$ \\
\hline \multirow[t]{4}{*}{ Family } & Achnanthaceae & & & & & & & & & \\
\hline & Achnanthes convergens & & & & & & + & & & W (Chung 1993) \\
\hline & A. inflata & & & & & & & & + & $\begin{array}{l}\text { W (Chung 1993), R (Lowe et al. } \\
\text { 2009) }\end{array}$ \\
\hline & A. minutissima & & & & & & + & & & $\begin{array}{l}\text { W (Chung 1993), R (Poulíčková } \\
\text { and Hašler 2007) }\end{array}$ \\
\hline \multirow[t]{3}{*}{ Family } & Nitzschiaceae & & & & & & & & & \\
\hline & Nitzschia frustulum & & & & & + & & & & W (Chung 1993), R (Hyvert 1972) \\
\hline & Hantzschia amphioxys & & + & & ++ & ++ & & & +++ & $\begin{array}{l}\text { W (Chung 1993), R (Poulíčková } \\
\text { and Hašler 2007) }\end{array}$ \\
\hline Order & Centrales & & & & & & & & & \\
\hline Suborder & Coscinodiscineae & & & & & & & & & \\
\hline \multirow[t]{3}{*}{ Family } & Melosiraceae & & & & & & & & & \\
\hline & Melosira varians & & & & & & & + & + & $\begin{array}{l}\text { W (Chung 1993), R (Poulíčková } \\
\text { and Hašler 2007) }\end{array}$ \\
\hline & Orthoseira roeseana & & & & & & & & + & $\begin{array}{l}\text { W (Chung 1993), R (Chung 1993, } \\
\text { Poulíčková and Hašler 2007) }\end{array}$ \\
\hline Phylum & Chlorophyta & & & & & & & & & \\
\hline Class & Chlorophyceae & & & & & & & & & \\
\hline Order & Ulotrichales & & & & & & & & & \\
\hline Family & Ulotrichaceae & & & & & & & & & \\
\hline
\end{tabular}


Table 3. Continued

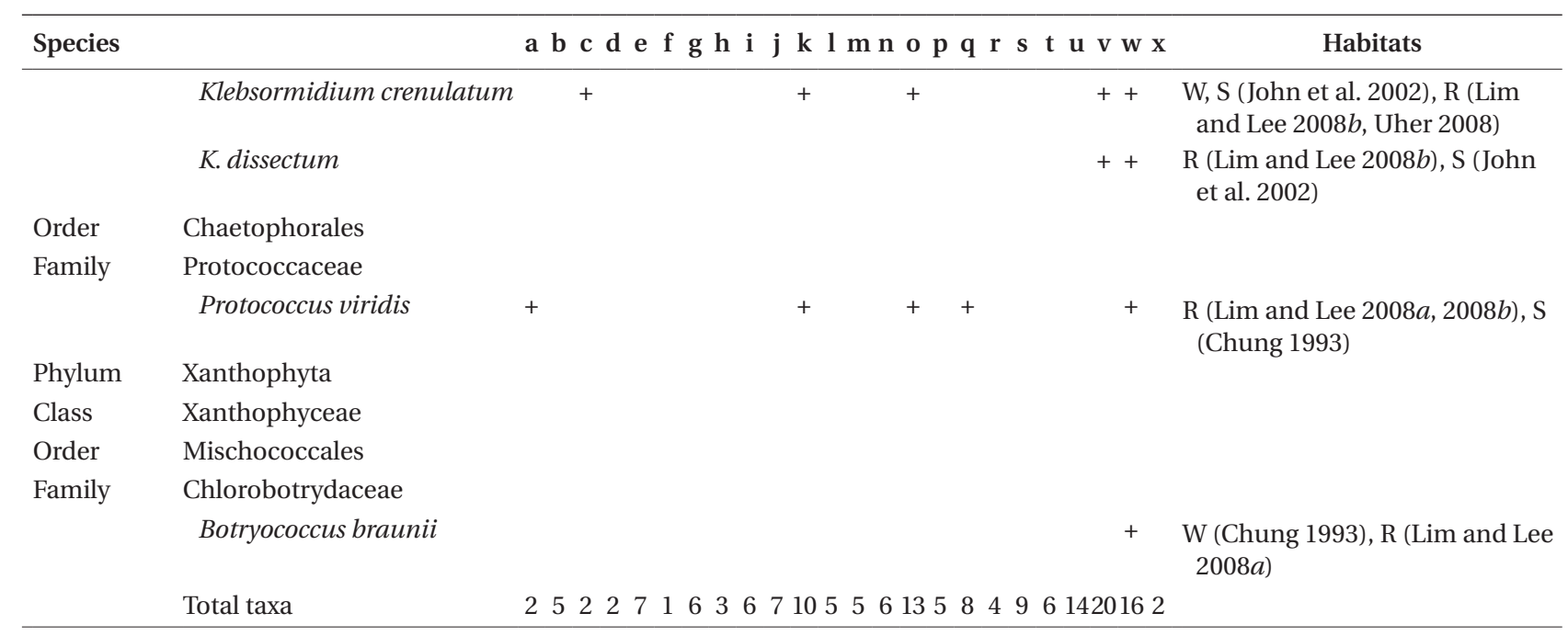

W, water; R, rock; S, soil; M, moss.

${ }^{a}$ Newly recorded species.

\section{Sites of collection. a, b, e, i, k, l, m, o, v, w (Figs 3C \& 4D). \\ Order Stigonematales \\ Family Stigonemataceae \\ Stigonema ocellatum (Dillwyne) Thuret f. ocellatum}

Cell shaped like grass or a thread; $25-50 \mu \mathrm{m}$ wide with irregular branching. Usually exists as a single filament, but occasionally can be found as double filaments. Branches are slightly slimmer than the main axis. Sheath is relatively thick, and may have yellow, yellow-brown, or no color. Trichomes are elliptical or square-shaped, and it inhabits humid dirt, rocks, and grass (Hirose et al. 1977).

Stigonema ocellatum $\mathrm{f}$. ocellatum has been studied on rocks exposed to natural light in Venezuela (Lakatos et al. 2001). In particular, it was found in places far away from water in the Australian study; however, we found it on stoneworks exposed to aerial algae without any relationship to water distance.

Sites of collection. c, e, g, h, I, j, l, m, r, s, t, u, v, w (Figs 3B \& 4E).

\section{Order Nostocales \\ Suborder Oscillatoriineae \\ Family Oscillatoriaceae \\ Oscillatoria boryana Kützing}

Cell dark colored; trichomes are flexible in the shape of a wave. The cell continuously folds, and length is less than or equal to half the width. Width of the cell is 6-8 $\mu \mathrm{m}$, and length is $4-6 \mu \mathrm{m}$. The apical cell is circular or a sharp circular cone, and has no calyptra (Hirose et al. 1977).

Oscillatoria boryana has been found in India (Kalavathi et al. 2001), and some studies have reported it in Japan, China, and North America (Hirose et al. 1977). We found it at one site, the Yeondae small temple A-3, with a tree distance of $0 \mathrm{~m}$, and a water distance of $>1,000 \mathrm{~m}$. These results indicates that it maintains humidity using trees.

Site of collection. $r$ (Figs 3D \& 4B).

Cyanophytes were dominant at 22 sites among the aerial algae (Table 4). In particular, cyanophytes dominated by $>50 \%$ at 15 sites, including Gwang-gyo mountain 1-1 (Kim et al. 2010). Hantzschia amphioxys (HAAM) and Cymbella delicatula, which are bacillariophytes were dominant at the Yeondae small temples A-1 and A-2. HAAM is known to be found at high humidity sites with bryophytes (Lim and Lee 2008a, Kim et al. 2010). Filamentous cyanophytes of Scytonema, Stigonema, Oscillatoria, and Nostoc were dominant at 15 sites including Gwang-gyo Mountain 1-1. Coccoid-shaped cyanophytes such as Chroococcus and Aphanocapsa were dominant at seven sites including Gwang-gyo Mountain 1-2.

A principal component analysis was conducted to identify the relationships between the aerial algae and ecological factors. The varimax method (Kim et al. 2007) was used to obtain the results (Fig. 5).

All species were divided into ecological groups according to the correlation coefficients. The identified species were divided into biological pollution coverage of bryophyte (Br), high humidity (Hu), and dry (Dry) groups with 

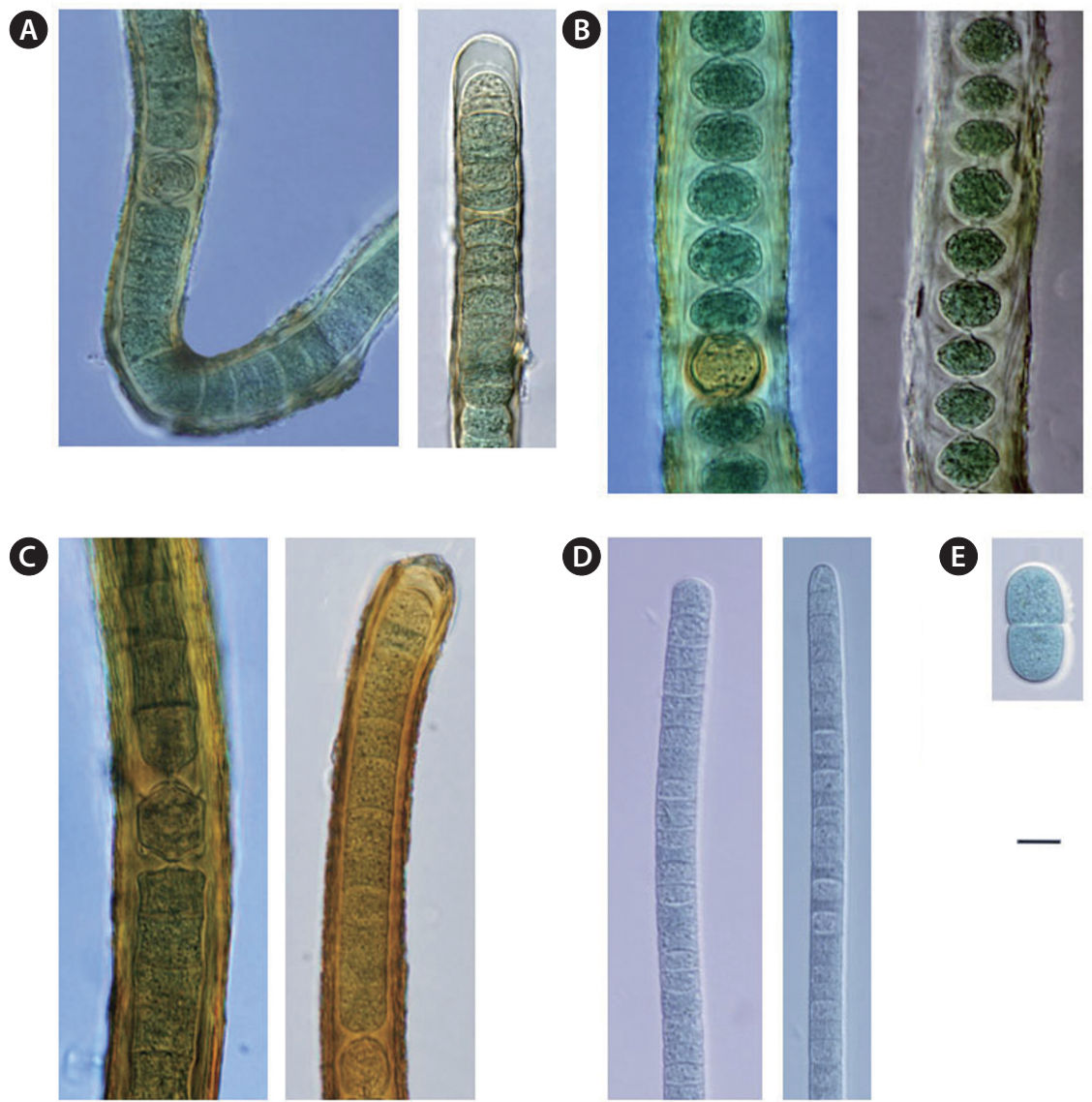

Fig. 3. Microscopic photographs of newly recorded species in Korea found at 24 stoneworks from 2009 to 2010. (A) Scytonema coactile var. thermalis. (B) Stigonema ocellatum f. ocellatum. (C) Scytonema coactile var. minor. (D) Oscillatoria boryana. (E) Synechococcus aeruginosus. Scale bar represents: $\mathrm{A}-\mathrm{E}, 10 \mu \mathrm{m}$.

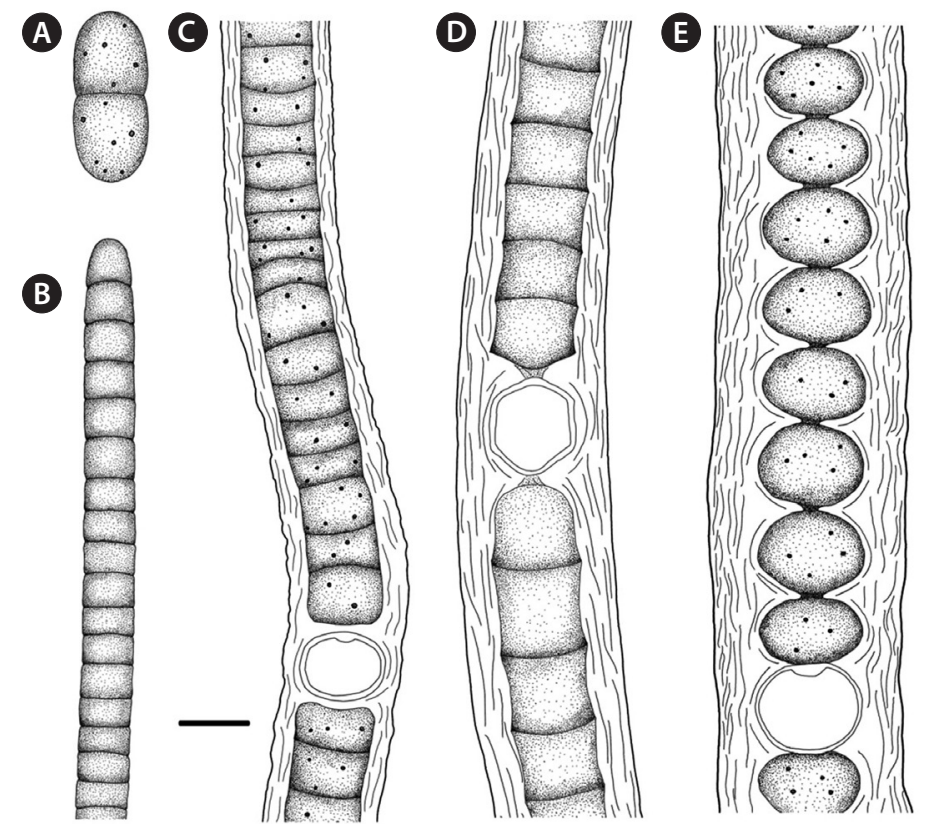

Fig. 4. Illustrations of newly recorded species in Korea found at 24 stoneworks from 2009 to 2010. (A) Synechococcus aerugonosus. (B) Oscillatoria boryana. (C) Scytonema coactile var. thermalis. (D) Scytonema coactile var. minor. (E) Stigonema ocellatum f. ocellatum. Scale bars represents: A-E, $10 \mu \mathrm{m}$. 


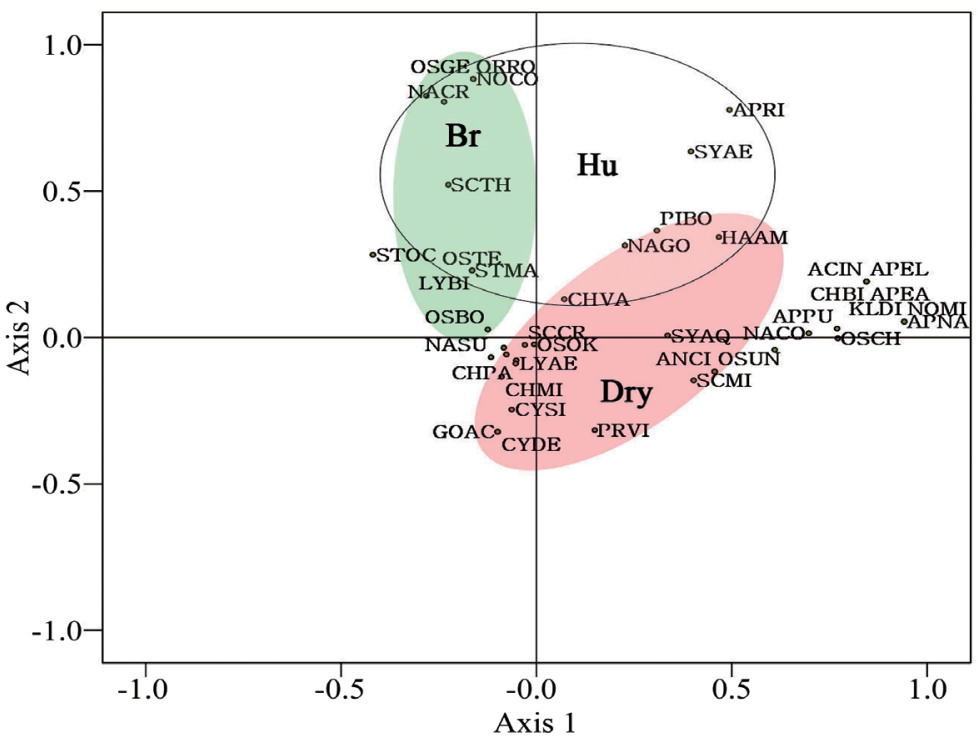

Fig. 5. Principal component analysis plot of aerial algae from 24 stoneworks in Korea during 2009 to 2010. Br, bryophyte coverage; Hu, humidity; Dry, negative correlation between humidity and canopy. ACIN, Achnanthes inflata; APEA, Aphanocapsa eachista; ANCl, Anabaena circinalis; APEL, Aphanocapsa elachista var. plantorica; APNA, Aphanothece naegelii; APPU, Aphanocapsa pulchra; APRI, Aphanocapsa rivularis; CHBI, Chroococcus bituminosus; CHMI, Chroococcus minutus; CHPA, Chroococcus pallidus; CHVA, Chroococcus varius; CYDE, Cymbella delicatula; CYSI, Cymbella sillesiaca; GOAC, Gomphonema acuminatum; HAAM, Hantzschia amphioxys; KLDI, Klebsormidium dissectum; LYAE, Lyngbya aerugineocoerulea; LYBI, Lyngbya birgei; NACO, Navicula contenta; NACR, Navicula crytocephala; NAGO, Navicula goeppertiana; NASU, Navicula subminuscula; NOCO, Nostoc commune; NOMI, Nostoc microscopicum; ORRO, Orthoceira roeseana; OSBO, Oscillatoria boryana; OSCH, Oscillatoria chlorina; OSGE, Oscillatoria geminata var. subphurea; OSOK, Oscillatoria okeni; OSTE, Oscillatoria terebriformis; OSUN, Oscillatoria uncinata; PIBO, Pinnularia borealis; PRVI, Protococcus viridis; SCCR, Scytonema crispum; SCMI, Scytonema coactile var. minor; SCTH, Scytonema coactile var. thermalis; STMA, Stigonema mamillosum; STOC, Stigonema ocellatum; SYAE, Synechococcus aeruginosus; SYAQ, Synechocystis aquatilis.

Table 4. Dominant species at 24 stoneworks in Korea during March 2009 to October 2010

\begin{tabular}{clc}
\hline Site & \multicolumn{1}{c}{ Dominant species } & $\mathbf{\%}$ \\
\hline $\mathrm{a}$ & Scytonema coactile var. minor & 89.6 \\
$\mathrm{~b}$ & Chroococcus varius & 61.8 \\
$\mathrm{c}$ & Stigonema ocellatum f. ocellatum & 71.1 \\
$\mathrm{~d}$ & Oscillatoria chlorine & 75.0 \\
$\mathrm{e}$ & Stigonema ocellatum $\mathrm{f}$. ocellatum & 62.3 \\
$\mathrm{f}$ & Chroococcus varius & 98.0 \\
$\mathrm{~g}$ & Oscillatoria limosa & 53.1 \\
$\mathrm{~h}$ & Chroococcus varius & 47.2 \\
$\mathrm{i}$ & Scytonema coactile var. minor & 53.2 \\
$\mathrm{j}$ & Scytonema coactile var. minor & 81.1 \\
$\mathrm{k}$ & Chroococcus minutes & 42.3 \\
$\mathrm{l}$ & Stigonema ocellatum $\mathrm{f}$ ocellatum & 77.6 \\
$\mathrm{~m}$ & Scytonema coactile var. minor & 43.7 \\
$\mathrm{n}$ & Chroococcus varius & 57.6 \\
$\mathrm{o}$ & Scytonema coactile var. minor & 44.7 \\
$\mathrm{p}$ & Hantzschia amphioxys & 49.8 \\
$\mathrm{q}$ & Cymbella delicatula & 44.0 \\
$\mathrm{r}$ & Scytonema coactile var. thermalis & 65.4 \\
$\mathrm{~s}$ & Stigonema mamillosum & 31.2 \\
$\mathrm{t}$ & Aphanocapsa pulchra & 72.4 \\
$\mathrm{u}$ & Nostoc commune & 96.0 \\
$\mathrm{v}$ & Oscillatoria chlorina & 38.0 \\
$\mathrm{w}$ & Oscillatoria chlorina & 22.1 \\
$\mathrm{x}$ & Aphanocapsa grevillei & 89.1 \\
\hline & &
\end{tabular}

a negative correlation with humidity and canopy (Fig. 5). Species in the Br group were cyanophytes and bacillariophytes such as Nostoc commune (NOCO), Oscillatoria geminata var. subphurea (OSGE), Orthoceira roeseana (ORRO) and Navicula crytocephala (NACR). These species showed high correlation coefficients with bryophyte biological pollution coverage ranging from 0.68 to 0.84 . The Dry included Chroococcus pallidus, HAAM, SCMI among others. The Hu group included CHVA, SCTH, Pinnularia borealis (PIBO), Navicula goeppertiana (NAGO), and SYAE.

\section{DISCUSSION}

Physico-chemical environmental factors vary greatly due to seasonal differences and the weather conditions on the date of collection. This seems to affect changes in aerial algae groups inhabiting stoneworks (Lim and Lee $2008 a$ ). Korean aerial algae now include total 108 taxa by adding 5 taxa from the present study. We found the following ten species which are aquatic species found in aerial conditions: Aphanocapsa rivularis, SCMI, SCTH, Oscillatoria boryana, OSGE, O. iwanoffiana, Gompho- 
Table 5. Analysis of the correlations between environmental factors in the 24 stoneworks in Korea collected during March 2009 to October 2010

\begin{tabular}{llllllllc}
\hline & Cov & Can & TD & WD & Tp & STp & Hu & L \\
\hline Cov & 1.00 & 0.31 & $-0.79^{* *}$ & -0.30 & $0.60^{* *}$ & $0.50^{*}$ & $0.55^{*}$ & 0.06 \\
Can & & 1.00 & -0.37 & -0.18 & $0.71^{* *}$ & $0.68^{* *}$ & 0.09 & 0.17 \\
TD & & & 1.00 & 0.10 & $-0.62^{* *}$ & $-0.55^{* *}$ & $-0.84^{* *}$ & -0.22 \\
WD & & & & 1.00 & -0.01 & 0.06 & $0.57^{*}$ & $0.54^{*}$ \\
Tp & & & & 1.00 & $0.97^{* *}$ & $0.72^{* *}$ & $0.50^{*}$ \\
STp & & & & & & & $0.60^{*}$ & $0.66^{* *}$ \\
Hu & & & & & & & 1.00 & 0.21 \\
Lux & & & & & & & & \\
\hline
\end{tabular}

The results of the correlation analysis between environmental factors showed that humidity had significant correlations with tree distance, water distance, and plant coverage with values of $-0.84,0.57$ and 0.55 , respectively. Moreover, light intensity and water distance were correlated (correlation coefficient $=0.54$ ).

Cov, plant coverage; Can, canopy; TD, tree distance; WD, water distance; Tp, temperature; STp, surface temperature; Hu, humidity; L, light intensity. " $p<0.05,{ }^{* *} p<0.01, n=24$.

nema acuminatum, Navicula subminuscula, Achnanthes convergens, and Cymbella delicatula (Table 3). Species in the Br group were cyanophytes and bacillariophytes such as NOCO, OSGE, ORRO, and NACR. Algae in the genus Nostoc live mainly in terrestrial habitats and appear to be attached to lichen, fungi, bryophytes, and vascular plants (Rai et al. 2002). The Br group plays an important role in habits of aerial algae by providing humid conditions. PIBO, NAGO, HAAM of the Dry and Hu groups were classified as aerial diatoms and are found under conditions with $80 \%$ humidity (Uher 2008), and also in conditions of $40 \%$ humidity and high light (Kim et al. 2011); therefore, it was thought that these aerial algae live in a wide range of conditions.

Correlations among other environmental factors were tested to identify other environmental factors that could substitute for humidity and light intensity (Table 5). As a result, tree distance, water distance, and plant coverage were replaceable environmental factors.

\section{ACKNOWLEDGEMENTS}

This research was supported by the foundation research project (2011-0005974) of National Research Foundation of Korea and National Research Institute of Cultural Heritage (NRICH-1107-B01F-1).

\section{REFERENCES}

Allsopp, D., Seal, K. \& Gaylard, C. 2004. Introduction to biodeterioration. 2nd ed. Cambridge University Press, Cambridge, $237 \mathrm{pp}$.
Badawy, M. I., Abou-Waly, H. F. \& Ali, G. H. 1999. Excretion products of algae and their occurrence in Solar Lake. Taba, Egypt. Int. J. Environ. Health Res. 9:233-243.

Bellinzoni, A. M., Caneva, G. \& Ricci, S. 2003. Ecological trends in travertine colonisation by pioneer algae and plant communities. Int. Biodeterior. Biodegrad. 51:203210.

Cavacini, P. 2001. Soil algae from northern Victoria Land (Antarctica). Polar Biosci. 14:45-60.

Chang, Y. K., Lee, J. W., Jeon, S. L. \& Kim, M. R. 1998. A taxonomic study of the soil algae in Korea. J. Nat. Sci. Sookmyung Women's Univ. Korea 9:39-43.

Chung, J. 1993. Illustration of the freshwater algae of Korea. Academy Publ. Co., Seoul, 496 pp.

Chung, Y. H. 1979. The basic study for the ecology of fungi and algae for the conservation of soil environments. Rep. Conserv. Nat. 1:1-395.

Crispim, C. A., Gaylarde, C. C. \& Gaylarde, P. M. 2004. Biofilms on church walls in Porto Alegre, RS, Brazil, with special attention to cyanobacteria. Int. Biodeterior. Biodegrad. 54:121-124.

Ghadai, A. K., Sahoo, S. \& Raut, S. 2010. Agroecological survey of cyanobacterial population in paddy field soils of Gunupur. Int. J. Agric. Sci. 2:28-32.

Gomez, S. R., Johansen, J. R. \& Lowe, R. L. 2003. Epilithic aerial algae of Great Smoky Mountains national park. Biologia (Bratisl.) 58:603-615.

Hirose, H. M., Akiyama, T., Imahori, H., Kasaki, H., Kumano, S., Kobayasi, H., Takahashi, E., Tsumura, T., Hirano, M. \& Yamagishi, T. 1977. Illustrations of the Japanese freshwater algae. Uchidarokakugo Publishing Co., Ltd., Tokyo, $933 \mathrm{pp}$.

Hyvert, G. 1972. The conservation of Borobudur Temple. Unesco, Jakarta, 172 pp. 
John, D. M., Whitton, B. A. \& Brook, A. J. 2002. The freshwater algal flora of the British Isles. Cambridge University Press, Cambridge, 702 pp.

Kalavathi, D. F., Uma, L. \& Subramanian, G. 2001. Degradation and metabolization of the pigment: melanoidin in distillery effluent by the marine cyanobacterium Oscillatoria boryana BDU 92181. Enzyme Microb. Technol. 29:246-251.

Kim, G. H., Klotchkova, T. A. \& Suh, M. C. 2001. The effect of chemical treatments on biodeterioration of stone cultural properties. Korean J. Environ. Biol. 19:101-105.

Kim, K. A., Kim, Y. J., Song, M. J., Ji, K. W., Yu, P. J. \& Kim, C. W. 2007. Evaluation of water quality characteristics in the Nakdong River using multivariate analysis. J. Korean Soc. Water Qual. 23:814-821.

Kim, Y. J., Kim, O. J. \& Lee, O. M. 2010. The distribution of aerial algae and the assessment of biological pollution class at 8 stone cultural heritages in Korea. J. Conserv. Sci. 26:259-268.

Kim, Y. J., Kim, O. J. \& Lee, O. M. 2011. The community of aerial algae and the biological pollution coverage at 9 stone cultural heritages in Korea. J. Conserv. Sci. 27:145-154.

Klochkova, T. A. \& Kim, G. H. 2005. Ornamented resting spores of a green alga, Chlorella sp., collected from the stone standing buddha statue at Jungwon Miruksazi in Korea. Algae 20:295-298.

Lakatos, M., Bilger, W. \& Büdel, B. 2001. Carotenoid composition of terrestrial Cyanobacteria : response to natural light conditions in open rock habitats in Venezuela. Eur. J. Phycol. 36:367-375.

Lamenti, G., Tiano, P. \& Tomaselli, L. 2000. Biodeterioration of ornamental marble statues in the Boboli Gardens (Florence, Italy). J. Appl. Phycol. 12:427-433.

Lim, A. S. \& Lee, O. M. $2008 a$. Distribution of aerial algae and biological classes in five stone cultural properties of Korea. Algae 23:63-69.

Lim, A. S. \& Lee, O. M. 2008b. The distribution of aerial algae and the evaluation of algal inhabitation on five stone cultural properties in Gyeonggi-do. Algae 23:269-276.

Lowe, R. L., Sherwood, A. R. \& Ress, J. A. 2009. Freshwater species of Achnanthes Bory from Hawaii. Diatom Res. 24:327-340.

National Research Institute of Cultural Heritage. 2008. Development on evaluation technology of deterioration for conservation of stone cultural properties. Research report. National Research Institute of Cultural Heritage, Daejeon, 652 pp.
Naz, S., Hasan, M. U. \& Shameel, M. 2004a. Biodiversity of Oscillatoria (Nostocophyceae, Cyanophyta) from northern areas of Pakistan. Pak. J. Bot. 36:503-530.

Naz, S., Hasna, M. U. \& Shameel, M. 2004b. Taxonomic study of Chroocophyceae (Cyanophyta) from northern areas of Pakistan. Pak. J. Bot. 36:247-281.

Poulíčková, A. \& Hašler, P. 2007. Aerophytic diatoms from caves in central Moravia (Czech Republic). Preslia 79:185-204.

Prescott, G. W. 1973. Algae of the western great lakes area. Otto Koeltz Science Publishers, Koenigstein, 660 pp.

Prescott, G. W., Bicudo, C. E. M. \& Vinyard, W. C. 1982. A synopsis of North American Desmids. Part II. Section 4. The University of Nebraska Press, Lincoln, NE, 358 pp.

Prescott, G. W., Croasdale, H. T. \& Vinyard, W. C. 1972. North American flora, Series II. Part 6. Desmidiales. New York Botanical Garden, New York, 37 pp.

Prescott, G. W., Croasdale, H. T. \& Vinyard, W. C. 1977. A synopsis of North American Desmids. Part II. Desmidiaceae: Placodermae. Section 2. The University of Nebraska Press, Lincoln, NE, 207 pp.

Prescott, G. W., Croasdale, H. T., Vinyard, W. C. \& Bicudo, C. E. M. 1981. A synopsis of North American Desmids. Part II. Section 3. The University of Nebraska Press, Lincoln, NE, 385 pp.

Rai, A. N., Bergman, B. \& Rasmussen, U. 2002. Cyanobacteria in symbiosis. Kluwer Academic Publishers, Dordrecht, $359 \mathrm{pp}$.

Sarró, M. I., García, A. M., Rivalta, V. M., Moreno, D. A. \& Arroyo, I. 2006. Biodeterioration of the Lions fountain at the Alhambra Palace, Granada (Spain). Build. Environ. 41:1811-1820.

Tiwari, G. L. 1972. A study of the blue-green algae from paddy field soils of India. Hydrobiologia 39:335-350.

Uher, B. 2008. Spatial distribution of cyanobacteria and algae from the tombstone in a historic cemetery in Bratislava, Slovakia. Fottea 9:81-92.

Videla, H. A., Guiamet, P. S. \& Gomez de Saravia, S. 2000. Biodeterioration of Mayan archaeological sites in the Yucatan Peninsula, Mexico. Int. Biodeterior. Biodegrad. 46:335-341.

Wehr, J. D., \& Sheath, R. G. 2003. Freshwater algae of North America: ecology and classification. Academic Press, San Diego, CA, 918 pp.

West, G. S. \& Fritsch, F. E. 1972. A treatise on the British Freshwater Algae. Cambridge University Press, Cambridge, $534 \mathrm{pp}$. 